\title{
Polymerase Chain Reaction as a Useful and Simple Tool for Rapid Diagnosis of Tuberculous Meningitis in a Brazilian Tertiary Care Hospital
}

\author{
José Miguel Dora', Guilherme Geib ${ }^{1}$, Rafael Chakr ${ }^{1}$, Fernanda de Paris², Alice Beatriz Mombach' \\ Larissa Lutz ${ }^{2}$, Carolina Fischinger Moura de Souza ${ }^{2}$ and Luciano Z. Goldani ${ }^{3}$ \\ ${ }^{1}$ Internal Medicine Section; ${ }^{2}$ Clinical Pathology Section; ${ }^{3}$ Infectious Diseases Unit; Clinical Hospital of Porto Alegre, \\ Federal University of Rio Grande do Sul Porto Alegre, RS, Brazil
}

\begin{abstract}
Meningitis is a severe and potentially fatal form of tuberculosis. The diagnostic workup involves detection of acid-fast bacilli (AFB) in the cerebrospinal fluid (CSF) by microscopy or culture, however, the difficulty in detecting the organism poses a challenge to diagnosis. The use of the polymerase chain reaction (PCR) in the diagnostic approach to Mycobacterium tuberculosis (MTB) meningitis has been reported as a fast and accurate method, with several commercial kits available. As an alternative, some institutions have been developing inexpensive in house assays. In our institution, we use an in house PCR for tuberculosis. We analyzed the performance of our PCR for the diagnosis of MTB meningitis in 148 consecutive patients, using MTB culture as gold standard. The sensitivity and specificity of CSF PCR for the diagnosis of MTB meningitis was $50 \%$ and $98.6 \%$ respectively with a concordance with CSF mycobacterial culture of $96 \%($ Kappa=0.52). In contrast to CSF cultures for MTB, our PCR test is a fast, simple and inexpensive tool to diagnose tuberculous meningitis with a performance similar to that obtained with the available commercial kits. Key-Words: Tuberculous meningitis, diagnosis, polymerase chain reaction, PCR, Mycobacterium tuberculosis.
\end{abstract}

Tuberculous meningitis is a potentially fatal form of tuberculosis, with serious long-term consequences [1]. Confirmation of the diagnosis is challenging. The traditional approach of clinical data with added biochemical and cytological findings of the cerebrospinal fluid (CSF) cannot confirm or exclude the condition [2,3]. Typical results include lymphocytic pleocytosis, elevated protein and low glucose contents. The diagnostic workup also involves detection of acid-fast bacilli (AFB) in the CSF by microscopy and culture. Due to the paucibacillary characteristic of this condition, the sensitivity of these tests is low [3,4]. Collecting large amounts of CSF (30mL) may provide better diagnostic results [4]. Some studies indicate that an elevated CSF adenosine deaminase (ADA) level may support the diagnosis in a proper clinical setting, but the cut off value is not clear and additional studies are necessary [5].

The use of the polymerase chain reaction (PCR) to amplify specific regions of the Mycobacterium tuberculosis (MTB) genome emerged as a tool for rapid and accurate diagnosis of tuberculous meningitis. The performance of this test has been studied since 1990, with results disclosing high specificity and variable sensitivity [6-9]. The exact role of this test in the diagnostic approach remains controversial. Its clinical usefulness appears to be in patients with smear negative CSF for AFB, which occurs in the majority of patients with tuberculous meningitis [3].

Received on 9 December 2007; revised 17 March 2008.

Address for correspondence: Dr Luciano Z. Goldani. Serviço de Medicina Interna. Hospital de Clínicas de Porto Alegre. Rua Ramiro Barcelos 2350. Zip code: 90035-002. Porto alegre, RS, Brazil. E-mail: lgoldani@ufrgs.br. Phone: 55-51-2101.8152. This is study was supported in part by CNPq (Brazilian National Council for Research).

The Brazilian Journal of Infectious Diseases 2008;12(3):245-247. (C) 2008 by The Brazilian Journal of Infectious Diseases and Contexto Publishing. All rights reserved.
Considering that results of the different in house MTB PCR assays are heterogeneous, it is not possible to extrapolate data from one institution to another. In our institution, a tertiarycare teaching hospital, we use an in house assay that was not previously validated.

We conducted a study to analyze the accuracy of our PCR in diagnosing tuberculous meningitis.

\section{Material and Methods}

A transversal study was conducted between January, 2005 and May, 2006 at Hospital de Clínicas de Porto Alegre located in southern Brazil. All patients with meningitis where a cerebrospinal fluid MTB PCR and MTB cultures were ordered were included in the study. For patients where more than one MTB PCR was ordered, only the results on the first sample were considered in the analysis. Additional laboratory tests and imaging studies, such as head CT scan, cryptococcal antigen test, CSF bacterial and fungal cultures, toxoplasma and syphilis serology, were also reviewed for alternative diagnosis of the meningitis,

The CSF samples were initially submitted to a nested PCR with primers to 65-KDa antigen, common to several pathogenic mycobacteria. The first nested PCR was done with the external primers 5' GCC AAG AAG ACC GAT GAC GT 3' and 5'TCG TTG CCC ACC TTG TCC AT 3' that amplify a DNA fragment of 280 base pairs (bp). In the second reaction, the amplicon of the first DNA reaction was amplified to a DNA fragment of 142 bp, using the internal primers 5' CCA ACC CGC TCG GTC TCAA3' and 5' CCG ATG GAC TGG TCA CCC 3'. In those samples which Mycobacterium sp. DNA was amplified, a second nested PCR was done. For the amplification of Mycobacterium tuberculosis complex DNA, external primers TB290 5' GGC GGG ACA ACG CCG AAT TGC GAA 3' and TB856 5' CGA GCG TAG GCG TCG GTG ACAAAG 3' were used to generate a fragment of $600 \mathrm{bp}$. In the second reaction, the amplicon of the first DNA reaction was amplified to a DNA 
fragment of $170 \mathrm{bp}$, using the internal primers TB500 5' TAC TAC GAC CAGATC 3' and TB607 5'TTG GTGATC AGC CGT 3' [9]. Negative (distilled water) and positive controls (H37RV culture of Mycobacterium) were used for all reactions. Visualization of the amplification products was done using $10 \mu \mathrm{L}$ of the final volume in $0.5 \mu \mathrm{g} / \mathrm{mL}$ ethidium bromide $2 \%$ agarose gel electrophoresis under ultraviolet light.

All clinical specimens submitted for mycobacterial culture were processed and simultaneously cultured in BACTEC broth medium (Becton Dickinson, Sparks, Md.) and LowensteinJensen (LJ) solid medium. The BACTEC broth medium (12B) contains palmitic acid labeled with ${ }^{14} \mathrm{C}$; the mycobacteria catabolize the ${ }^{14} \mathrm{C}$-labeled palmitic acid and release ${ }^{14} \mathrm{CO}_{2}$; the ${ }^{14} \mathrm{CO}_{2}$ in the headspace gas is measured by an automated radiometric system (BACTEC 460 TB). The characteristic mycobacteria colonial morphology was evaluated on LJ and acid-fast bacilli (AFB) smears were also performed on the sediments. Differentiation of mycobacterial isolates such as Mycobacterium tuberculosis (MTB) or Mycobacterium sp. other than tuberculosis (MOTT) was based on the BACTEC NAP test. Positive control standard culture of Mycobacterium tuberculosis H37RV was processed with every batch of specimens.

A two by two table was created using the results obtained, with sensitivity, specificity, likelihood ratios and predictive values derived from it. Intertest agreement was evaluated by concordance and Kappa value. Statistical analysis was done using the software Statistical Package for the Social Sciences 13.0 (SPSS, Inc., Chicago, IL).

\section{Results}

A total of 148 patients were included in the study. The mean age of the patients was 38 and $60 \%$ of the patients were male. In addition, $72 \%$ of the patients were HIV-positive. Positive CSF cultures and PCR for MTB were found in $4.0 \%$ and 3.3\% respectively of the patients (Table 1 ). Three of 6 patients with positive CSF cultures for MTB had a negative CSF PCR for MTB. In addition, 2 of 5 patients with positive CSF PCR for MTB had negative CSF cultures for MTB. All patients with positive CSF cultures or PCR for MTB were considered to have MTB meningitis and received specific treatment. Alternative diagnosis for patients with negative CSF PCR and cultures for MTB included cryptococcal meningitis (15\%), central nervous toxoplasmosis (14\%), viral meningitis (9\%), neurosyphilis (4\%), and bacterial meningitis (4\%).

As shown in Table 1, the performance of CSF PCR for MTB was compared with CSF cultures for MTB. Despite its moderate sensitivity 0.50 (95\% CI 0.18-0.81), the CSF PCR assay for MTB detection had a high specificity of 0.98 (95\%CI 0.95-0.99) and a high negative predictive value of 0.97 (95\%CI 0.94-0.99).

\section{Discussion}

A large number of patients with the diagnosis of meningitis were included in our study, which reflects the scenario of a tertiary-care teaching hospital in southern Brazil with high attendance of a diverse population, including HIV-positive patients with multiple CNS syndromes mimicking tuberculous meningitis. This could explain the low prevalence of MTB meningitis among those with high clinical suspicion of the condition.

The high specificity of the CFS PCR for MTB (95\%-99\%) found in our study is in agreement with previously reported data and it was similar to CSF cultures (98\%) for MTB [3,6,7]. In contrast, the sensitivity of our PCR test to detect MTB was low. Potential explanations for the low sensitivity include a low bacillary load in the CSF, an inadequate quantity of CSF tested, or the presence of substances in the CSF that inhibit amplification. In a meta-analysis published by Pai M. et al. [4],

Table 1. CSF analysis of 148 patients with meningitis.

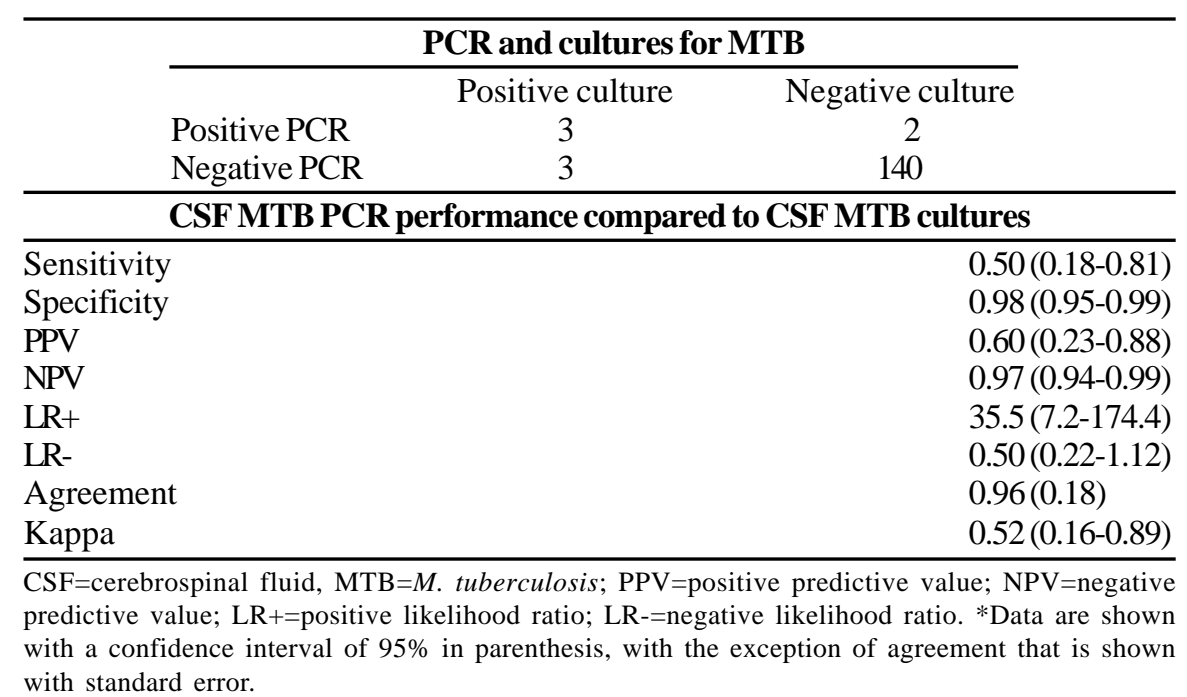


the sensitivity of commercial kits was of $56 \%$, similar to that found in the present study [4]. Considering those findings, a positive CSF PCR for MTB was virtually diagnostic of tuberculous meningitis in our patients [3]. Taking into consideration the low sensitivity of MTB culture and the length of time required for results, the PCR assists by saving time and increasing sensitivity for MTB diagnosis.

Nucleic amplification tests are not stand-alone tests and they are usually done along with conventional tests. However, we provide a simple, methodologically detailed, in house PCR technique that will help to improve the diagnosis of tuberculous meningitis. In contrast to commercial kits, in house techniques offer the advantage of lower costs, maintaining similar diagnostic performance. This is a very important issue in developing countries where tuberculosis is epidemic and few financial resources are available to public health.

\section{References}

1. World Health Organization. Global tuberculosis control. WHO Report, 2001. Geneva: World Health Organization 2001:18-9.
2. Hosoglu S., Ayaz C., Geyik M.F., Kokoglu O.F., Ceviz A. Tuberculous meningitis in adults: an eleven-year review. Int $\mathrm{J}$ Tuberc Lung Dis 1998;2:553-7.

3. Porkert M.T., Sotir M., Parrott-Moore P., Blumberg H.M. Tuberculous meningitis at a large inner-city medical center. Am J Med Sci 1997;313:325-31.

4. Pai M., Flores L.L., Pai N., et al. Diagnostic accuracy of nucleic acid amplification test for tuberculous meningitis: a systematic review and meta-analysis. Lancet Infect Dis 2003;3:633-43.

5. Thwaites G.E., Hien T.T. Tuberculous meningitis: too many questions, too few answers. Lancet Neurol 2005;4:160-70.

6. Ribera E., Martinez-Vazquez J.M., Ocana I., et al. Activity of adenosine deaminase in cerebrospinal fluid for the diagnosis and follow-up of tuberculous meningitis in adults. J Infect Dis 1987; 155:603-7.

7. Kaneko K., Onodera O., Miyatake T., Tsuji S. Rapid diagnosis of tuberculous meningitis by polymerase chain reaction (PCR). Neurology 1990;40:1617-8.

8. Folgueira L., Delgado R., Palenque E., Noriega A.R. Polymerase chain reaction for rapid diagnosis of tuberculous meningitis in AIDS patients. Neurology 1994;44:1336-8.

9. Kox L.F., Kuijper S., Kolk A.H. Early diagnosis of tuberculous meningitis by polymerase chain reaction. Neurology 1995; 45:2228-32. 